\title{
On the nature of lithium-rich giant stars
}

\section{Constraints from beryllium abundances ${ }^{\star}$}

\author{
C. H. F. Melo ${ }^{1}$, P. de Laverny ${ }^{2}$, N. C. Santos ${ }^{3,4}$, G. Israelian ${ }^{5}$, S. Randich ${ }^{6}$, \\ J. D. do Nascimento $\mathrm{Jr}^{7}$, and J. R. De Medeiros ${ }^{7}$ \\ ${ }^{1}$ European Southern Observatory, Casilla 19001, Santiago 19, Chile \\ e-mail: cmelo@eso.org \\ 2 Observatoire de la Côte d'Azur, Département Cassiopée, UMR 6202, BP 4229, 06304 Nice, France \\ 3 Centro de Astronomia e Astrofíca da Universidade de Lisboa, Observatório Astronómico de Lisboa, Tapada da Ajuda, \\ 1349-018 Lisboa, Portugal \\ ${ }^{4}$ Observatoire de Genève, 51 ch. des Maillettes, 1290 Sauverny, Switzerland \\ 5 Instituto de Astrofísica de Canarias, 38205 La Laguna, Tenerife, Spain \\ ${ }^{6}$ INAF/Osservatorio Astrofisico di Arcetri, Largo E. Fermi 5, 50125 Firenze, Italy \\ 7 Departamento de Física, Universidade Federal do Rio Grande do Norte, 59072-970 Natal, RN., Brazil
}

Received 5 August 2004 / Accepted 29 March 2005

\begin{abstract}
We have derived beryllium abundances for $7 \mathrm{Li}$-rich giant $(A(\mathrm{Li})>1.5)$ stars and 10 other Li-normal giants with the aim of investigating the origin of the lithium in the Li-rich giants. In particular, we test the predictions of the engulfment scenario proposed by Siess \& Livio (1999, MNRAS, 308, 1133), where the engulfment of a brown dwarf or one or more giant planets would lead to simultaneous enrichment of ${ }^{7} \mathrm{Li}$ and ${ }^{9} \mathrm{Be}$. We show that regardless of their nature, none of the stars studied in this paper were found to have detectable beryllium. Using simple dilution arguments we show that engulfment of an external object as the sole source of $\mathrm{Li}$ enrichment is ruled out by the $\mathrm{Li}$ and $\mathrm{Be}$ abundance data. The present results favor the idea that $\mathrm{Li}$ has been produced in the interior of the stars by a Cameron-Fowler process and brought up to the surface by an extra mixing mechanism.
\end{abstract}

Key words. stars: abundances - stars: interiors - stars: late-type

\section{Introduction}

The discovery of lithium excesses in a few low-mass giants in recent years represents one of the most exciting puzzles for stellar astrophysics. Essentially such stars show significantly higher lithium content than the values predicted in the framework of standard stellar evolution, some of them possessing surface lithium content approaching the present interstellar medium value of $A(\mathrm{Li}) \sim 3.0$ or even higher (de la Reza $\&$ da Silva 1995; Balachandran et al. 2000). Standard models predict that, after the first dredge-up and dilution due to the deepening of the convective zone, RGB stars should have a Li abundance 20-60 times below the initial value (e.g. Iben 1967). However, since low mass stars have partially destroyed their initial $\mathrm{Li}$ on the pre-main sequence (PMS) and on the main sequence (MS), the observed Li abundances in RGB stars are indeed much lower.

In spite of an increasing number of studies with a variety of propositions (see review by de la Reza 2000, and references

* Based on observations collected with the VLT/UT2 Kueyen telescope (Paranal Observatory, ESO, Chile) using the UVES spectrograph (program ID 69.D-0718A). therein), the root-cause of these highly abnormal abundances of Li in low-mass red giant stars remains unknown, adding a new critical question about these undoubtedly very complex physical systems. Some explanations are related to internal processes, such as a fresh lithium synthesis (e.g. Sackmann \& Boothroyd 1999), or a preservation of the initial lithium content (Fekel \& Balachandran 1993), whereas other explanations are based on external processes such as contamination of the stellar external layers by debris of nova ejecta or the engulfment of brown dwarfs or planets by the giant star (Alexander 1967; Brown et al. 1989; Gratton \& D’Antona 1989; Siess \& Livio 1999).

Particularly interesting have been those explanations based on fresh lithium production or planet engulfment, although recent observational studies have not resulted in any fully satisfactory conclusion. Sackmann and Boothroyd (1999) have shown that, under certain conditions, ${ }^{7} \mathrm{Li}$ can be created in low-mass red giants via the Cameron-Fowler mechanism, due to extra deep mixing and the associated cool bottom processing. Following these authors, this fresh material could account for the excess of lithium observed in the so-called lithium-rich giants. If deep circulation is a long-lived, continuous process, 
these lithium-rich stars should be completely devoid of beryllium and boron, whereas if it occurs in short-lived episodes, beryllium and boron might be only partially destroyed.

In the other context, theoretical predictions by Siess \& Livio (1999), which assume that a planet, brown dwarf, or very low mass star is dissipated at the bottom of the convective envelope of the giant star, point to several observational signatures that accompany the engulfing phenomenon. Among such signatures, there is the ejection of a shell and a subsequent phase of IR emission, an increase in the light elements surface content (specially in the ${ }^{7} \mathrm{Li}$, but also ${ }^{6} \mathrm{Li},{ }^{9} \mathrm{Be}$, and ${ }^{11} \mathrm{~B}$ ), potential stellar metallicity enrichment, spin-up of the star because of the deposition of orbital angular momentum, the possible generation of magnetic fields, and the related X-ray activity caused by the development of shear at the base of the convective envelope.

Concerning the light element enhancement, Israelian et al. (2001, 2003) used high-resolution and high signal-to-noise observations to show that ${ }^{6} \mathrm{Li}$ is present in the atmosphere of the extra-solar planet host star HD 82943. The authors interpreted the presence of ${ }^{6} \mathrm{Li}$ as evidence of a planet (or planets) having been engulfed by the parent star. Observations of Be II lines in two lithium-rich giants, HD 9746 and HD 112127, with the IUE indicated that $\mathrm{Be}$ is probably not preserved in these stars (De Medeiros et al. 1997). Such a result seems to indicate that $\mathrm{Be}$ and consequently the primordial Li were depleted in these two Li-rich stars. This suggests that additional Li was produced in the stellar interior or added from an external source. Castilho et al. (1999) have measured Be abundances for two Li-rich giants, concluding that, in such stars, Be is very depleted in relation to the initial Be abundance value of population I stars. In the present work we extend the work done in Castilho et al. by deriving Be abundances for all Li-rich giants visible in the southern hemisphere. Along with those $\mathrm{Li}$ abundances found in the literature, the newly derived Be abundances allow for a much stronger test of the engulfment scenario proposed by Siess \& Livio (1999), since the Li enrichment resulting from an engulfment episode would also be accompanied by a detectable Be enrichment.

\section{Sample and observations}

Approximately $1 \%$ of giant stars have anomalously high $\mathrm{Li}$ abundances compared to the values predicted by the standard models. Here we have selected 9 Li-rich stars that can be observed in the southern hemisphere along with another $10 \mathrm{Li}$ normal giants selected as a comparison sample.

Observations of the Be II doublet at $3131 \AA$ for the two groups were carried out in service mode using the UVES spectrograph (Dekker et al. 2000) attached to VLT/UT2 Kueyen telescope at ESO, Chile. UVES was operated in the blue-arm, which is equipped with an EEV $2048 \times 4102$ CCD. Using the cross-disperser \#1, the blue-arm covers the spectral range from $\sim 3020$ to $\sim 3880 \AA$. The spectral region around the Be II doublet is heavily crowded. Therefore, a spectral resolution $R$ higher than about 30000-40000 is mandatory (García López et al. 1995). In order to have a high signal-to-noise (around 100) and a high-resolution spectrum even during poor seeing conditions, the image slicer was inserted (Dekker et al. 2002). The obtained spectra have a resolution of around 70000 and an $S / N$ ratios about 70-80 in the region of the Be II doublet for all but three stars, which have a $S / N \sim 20-30$. Apparent visual magnitudes, $(B-V)$, parallaxes, and $A(\mathrm{Li})$ taken from the literature along with the obtained $S / N$ ratios are listed in Table 1 for both samples. The data collected was reduced using IRAF $^{1}$ (echelle package) to carry out the standard echelle spectrum reduction steps, namely, bias and background subtraction, flat-field correction, spectrum extraction and wavelength calibration. The wavelength calibration was done using a ThAr lamp spectrum as reference taken on the morning of the following day.

\section{Be abundance calculations}

\subsection{Stellar parameters}

For the program stars, effective temperatures were estimated from $(B-V)$ photometry found in the Hipparcos catalog (Perryman et al. 1997) and the calibrations of Alonso et al. (1999) and Houdashelt et al. (2000). We also considered $(b-y)$ colors, when available, from Hauck \& Mermilliod (1998) and the calibration of Alonso et al. (1999). In the color- $T_{\text {eff }}$ calibrations, we assumed a solar-metallicity for all the stars. For comparison, temperatures were derived with the different methods/colors in rather good agreement, implying that they should not be affected by too large systematic errors and that $[\mathrm{Fe} / \mathrm{H}]$ should not be significantly different from solar. Adopted effective temperatures are the ones computed using the $(B-V)-T_{\text {eff }}$ calibration of Alonso et al. (1999).

Parallax-based stellar gravities were estimated using parallaxes and visual magnitudes taken from the Hipparcos catalog with the bolometric corrections computed using the Flower (1996) $T_{\text {eff }}-B C$ calibration and effective temperatures computed as described above. Also, an $M_{v, \odot}=4.83$ and $B C_{\odot}=-0.12$ were adopted. Stellar masses were estimated by comparing the position of our sample stars in the colormagnitude diagram with the evolutionary tracks from Bertelli et al. (1994) computed using solar metallicity as shown in Fig. 1. Surface gravities were computed using Eq. (3) of Nissen et al. (1997).

In the spectral analysis, we used these parallax-based gravities when the error on the parallax was smaller than $15 \%$. For the remaining stars without a parallax or those whose mass could not be well determined (HD 176588), we assumed a gravity typical of giant stars $\log g=2.0$.

As for the metallicity, we considered mean values from the literature. For all the stars we also assumed a depthindependent microturbulence velocity of $2.0 \mathrm{~km} \mathrm{~s}^{-1}$ with an uncertainty of about $1.0 \mathrm{~km} \mathrm{~s}^{-1}$. A macro-turbulent velocity value of $2.0 \mathrm{~km} \mathrm{~s}^{-1}$ was also taken for all stars. Changing this latter value by $\pm 2 \mathrm{~km} \mathrm{~s}^{-1}$ would not change any of the results presented below. Rotational velocities from De Medeiros \& Mayor (1999) were considered, and $V_{\text {sini }}=1 \mathrm{~km} \mathrm{~s}^{-1}$ was used for the slower rotators.

${ }^{1}$ IRAF is distributed by National Optical Astronomy Observatories, operated by the Association of Universities for Research in Astronomy, Inc., under contract with the National Science Foundation, USA. 
Table 1. Sample stars and observation log.

\begin{tabular}{rccccc}
\hline \hline HD & $V$ & $(B-V)$ & $\pi$ (mas) & Night & $S / N$ at Be \\
\hline Li-rich giants: & & & & & \\
787 & 5.28 & 1.50 & 5.33 & $2002-07-30$ & 70 \\
19745 & 9.11 & 1.04 & - & $2002-07-30$ & 30 \\
30238 & 5.71 & 1.50 & 5.20 & $2002-09-10$ & 80 \\
39853 & 5.63 & 1.55 & 4.37 & $2002-09-09$ & 30 \\
95799 & 7.99 & 1.00 & - & $2002-05-21$ & 70 \\
176588 & 6.89 & 1.72 & 4.25 & $2002-06-01$ & 80 \\
183492 & 5.57 & 1.04 & 11.38 & $2002-06-02$ & 80 \\
217352 & 7.16 & 1.15 & 5.11 & $2002-07-31$ & 50 \\
219025 & 7.68 & 1.21 & 3.25 & $2002-07-29$ & 30 \\
\hline Li-normal stars: & & & & & \\
360 & 5.99 & 1.03 & 9.79 & $2002-08-04$ & 90 \\
1522 & 3.56 & 1.21 & 11.26 & $2002-08-04$ & 60 \\
4128 & 2.04 & 1.02 & 34.04 & $2002-08-04$ & 220 \\
5437 & 5.35 & 1.51 & 6.24 & $2002-09-10$ & 80 \\
61772 & 4.98 & 1.54 & 4.82 & $2002-04-18$ & 60 \\
61935 & 3.94 & 1.02 & 22.61 & $2002-04-18$ & 130 \\
95272 & 4.08 & 1.08 & 18.71 & $2002-04-18$ & 100 \\
105707 & 3.02 & 1.33 & 10.75 & $2002-04-29$ & 70 \\
126271 & 6.19 & 1.21 & 8.97 & $2002-05-31$ & 100 \\
220321 & 3.96 & 1.08 & 20.14 & $2002-06-13$ & 80 \\
\hline
\end{tabular}

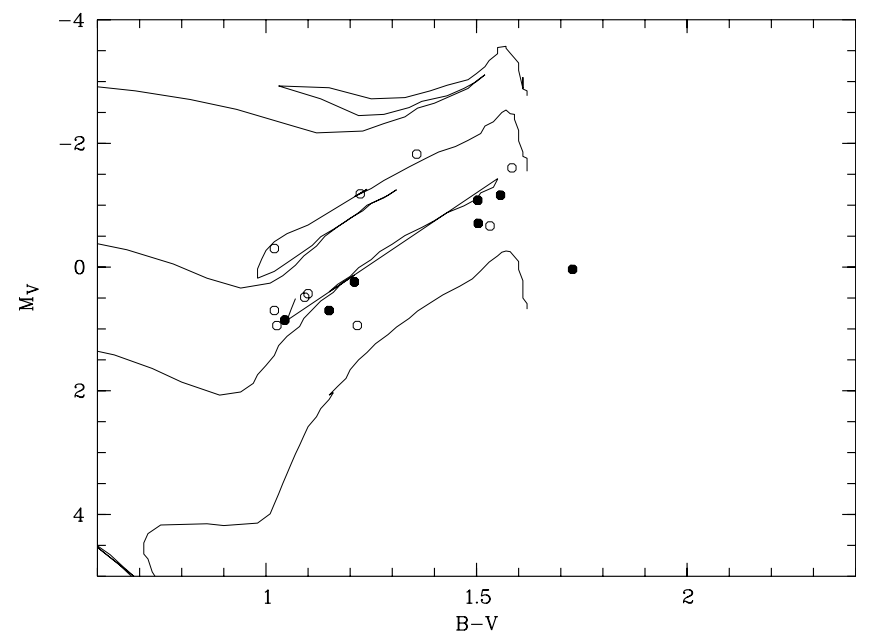

Fig. 1. Color-magnitude diagram of the observed sample. Li-normal and Li-rich giants are seen as heavy and light gray points, respectively. Overplotted are isochrones from Bertelli et al. (1994) for (from the bottom to top) log age $=10,9,8.6$, and 8 yr corresponding to masses on the RGB of 1, 1.9-1.95, and 4-5.3 solar masses.

Final values for the stellar masses, $T_{\text {eff }}$, and $\log g$ are given in Table 2. We estimate that the accuracy of our derived $T_{\text {eff }}$ and $\log g$ are, respectively, $\sim 100 \mathrm{~K}$ and 0.3 dex and about $1 \mathrm{~km} \mathrm{~s}^{-1}$ for the microturbulence.

\subsection{LTE chemical analysis of the program stars}

The chemical abundances were derived by fitting synthetic spectra to the observed profiles with the set of stellar parameters given in Table 2. LTE synthetic spectra were computed using a new version of the code MOOG written by
C. Sneden $(1973)^{2}$ and model atmospheres were interpolated in the grid of Kurucz (1993), ATLAS9.

We used the linelist provided by Garcia Lopez et al. (1995) specially built for solar-type stars. In order to test it for cooler stars as those studied in this work, we calibrated this linelist with the spectrum of Arcturus, a K1 giant with no detectable $\mathrm{Li}$ or Be on its surface. We further assumed the stellar parameters and abundances derived by Peterson et al. (1993). The oscillator strength of some lines between 3130 and $3132 \AA$ were corrected in order to better fit the Arcturus spectrum, assuming the abundances reported by Peterson et al. (1993). All the $g f$-values of the $\mathrm{OH}$ lines were decreased by $0.4 \mathrm{dex}$, and we adopted $g f=6 \times 10^{-2}$ for $\mathrm{CH} 3130.648, g f=0.1$ for $\mathrm{Cr} \mathrm{I}$ 3131.212 , and $g f=8.61 \times 10^{-3}$ for Co I 3131.825 . For the calibration of the Zr I 3131.109 and Mn I 3131.037 lines, we first derived the abundances of $\mathrm{Zr}$ and $\mathrm{Mn}$ from the Arcturus atlas using line data from Feltzing \& Gustafsson (1998). We found $[\mathrm{Zr} / \mathrm{Fe}]=-1.0$ and $[\mathrm{Mn} / \mathrm{Fe}]=-0.17$, and derived $g f$ values equal to $3.98 \times 10^{-2}$ and 0.296 , respectively. For some giant stars, it was also found that the Tm II 3131.255 may play an important role. But we were unable to derive the abundance of Tm in Arcturus. We therefore scaled the abundance of Tm with Fe (as done by Peterson et al. 1993) and checked that the $g f$-value of this line found in the original linelist leads to a good fit. Finally, it was found unnecessary to add any ad-hoc Fe I lines as proposed by Castilho et al. (1999) at $3130.995 \AA$ and Primas et al. (1997) at $3131.043 \AA$. The final spectral synthesis of Arcturus is shown in Fig. 2.

From the estimated stellar parameters, we first derived abundances of $\mathrm{O}, \mathrm{Mn}$, and $\mathrm{Tm}$ using the $\mathrm{OH}$ lines, the Mn I line at 3131.037 ̊ and Tm II line 3113.255 $\AA$. In a second iteration, the Be abundance was then determined. They are

\footnotetext{
${ }^{2}$ http://verdi.as.utexas.edu/moog.html
} 
Table 2. Atmospheric parameters and derived abundances for the program stars. Asterisks indicate guess values for the gravity or the metallicity. For stars with very large rotational velocities, we were unable to derive abundances of $\mathrm{O}, \mathrm{Mn}$, and Tm.

\begin{tabular}{|c|c|c|c|c|c|c|c|c|c|c|}
\hline HD & $T_{\text {eff }}(K)$ & $\log g$ & $M / M_{\odot}$ & {$[\mathrm{Fe} / \mathrm{H}]$} & $V_{\text {sini }}\left(\mathrm{km} \mathrm{s}^{-1}\right)$ & {$[\mathrm{O} / \mathrm{H}]$} & {$[\mathrm{Mn} / \mathrm{H}]$} & {$[\mathrm{Tm} / \mathrm{H}]$} & $\mathrm{A}(\mathrm{Be})^{\dagger}$ & $A(\mathrm{Li})$ \\
\hline \multicolumn{11}{|l|}{ Li-rich giants: } \\
\hline 787 & 3990 & 1.3 & 1.9 & +0.00 & 1.9 & +0.0 & -0.4 & -1.0 & no detection & $1.8^{a}$ \\
\hline 19745 & 4730 & $2.0(*)$ & & +0.10 & 1.0 & +0.6 & +0.3 & +0.5 & no detection & $4.1^{b}$ \\
\hline 30238 & 3990 & 1.4 & 1.9 & +0.00 & 1.0 & -0.2 & +0.0 & +0.0 & no detection & $0.8^{c}$ \\
\hline 39853 & 3920 & 1.1 & 1.6 & -0.40 & 1.0 & +0.0 & +0.0 & +0.0 & no detection & $2.8^{d}$ \\
\hline 95799 & 4800 & $2.0(*)$ & & -0.11 & 1.0 & +0.8 & +0.8 & +0.8 & no detection & $3.2^{e}$ \\
\hline 176588 & 3800 & $2.0(*)$ & & +0.00 & 1.0 & -0.2 & -0.3 & -0.4 & no detection & $1.1^{c}$ \\
\hline 183492 & 4720 & 2.6 & 2.0 & +0.00 & 1.0 & +0.6 & +0.6 & +1.1 & no detection & $2.0^{a}$ \\
\hline 217352 & \multirow{2}{*}{\multicolumn{10}{|c|}{$\begin{array}{l}\text { too large } V \sin i \\
\text { too large } V \sin i\end{array}$}} \\
\hline 219025 & & & & & & & & & & \\
\hline \multicolumn{11}{|l|}{ Li-normal stars: } \\
\hline 360 & 4750 & 2.7 & 2.1 & -0.20 & 1.0 & +0.5 & +0.2 & +1.0 & no detection & $0.2^{a}$ \\
\hline 1522 & 4400 & 1.8 & 3.2 & +0.20 & 1.0 & -0.3 & +0.0 & +0.0 & no detection & $<0.0^{a}$ \\
\hline 4128 & 4760 & 2.4 & 3.2 & +0.15 & 1.0 & -0.2 & +0.0 & +0.4 & no detection & $<0.2^{a}$ \\
\hline 5437 & 3950 & 1.3 & 1.4 & -0.20 & 1.0 & +0.2 & -0.2 & +0.0 & no detection & $0.1^{a}$ \\
\hline 61772 & 3880 & 1.0 & 1.9 & +0.10 & 1.0 & -0.2 & -0.4 & -0.4 & no detection & $-0.5:^{a}$ \\
\hline 61935 & 4760 & 2.7 & 2.4 & +0.00 & 1.0 & +0.2 & +0.3 & +1.0 & no detection & $0.2^{a}$ \\
\hline 95272 & 4620 & 2.4 & 2.1 & +0.00 & 1.0 & +0.4 & +0.2 & +0.9 & no detection & $<0.0^{a}$ \\
\hline 105707 & 4190 & 1.4 & 3.2 & +0.10 & 1.0 & +0.2 & +0.3 & +0.0 & no detection & $0.8^{a}$ \\
\hline 126271 & 4410 & 2.3 & 1.4 & +0.00 & 1.0 & +0.1 & +0.1 & +0.6 & no detection & $<-0.4^{a}$ \\
\hline 220321 & 4630 & 2.4 & 2.1 & -0.30 & 1.0 & +0.6 & +0.6 & +1.0 & no detection & $<-0.2^{a}$ \\
\hline
\end{tabular}

${ }^{a}$ Brown et al. (1989); ${ }^{b}$ de la Reza \& da Silva (1995); ${ }^{c}$ Castilho et al. (2000); ${ }^{d}$ Gratton \& D'Antona (1989); ${ }^{e}$ Luck (1994).

No detection is compatible with $A(\mathrm{Be})<-5$.

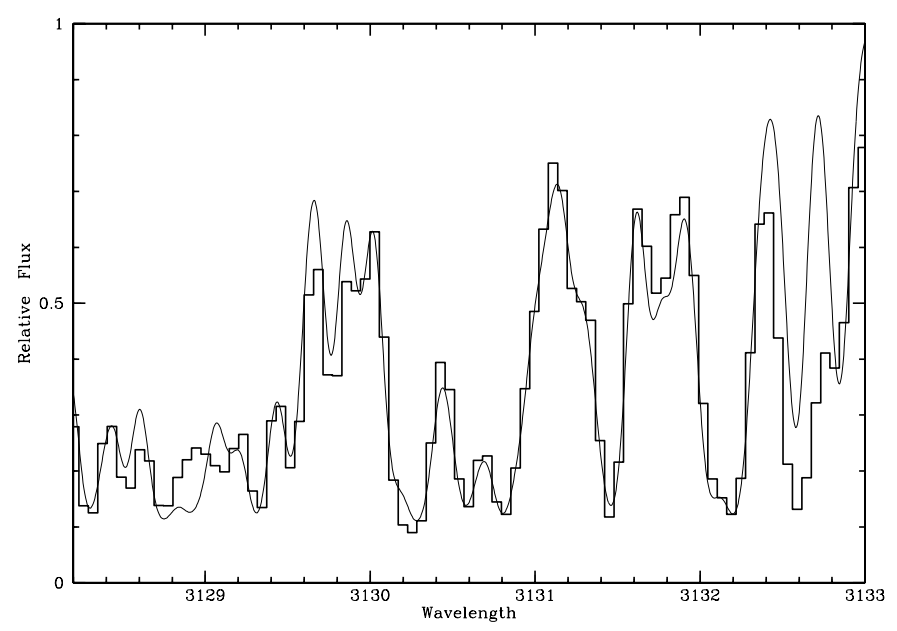

Fig. 2. Spectrum of Arcturus and its synthesis used in the calibration of the linelist provided by Garcia Lopez et al. (1995) specially for coolerstars. The oscillator strength of some lines between 3130 and $3132 \AA$ were corrected in order to better fit the Arcturus spectrum assuming the abundances reported by Peterson et al. (1993).

reported in Table 2. When comparing observations with our synthetic spectra, it was sometimes found necessary to add a small amount of veiling (about 10\%) to the synthetic spectra in order to improve the fits of the cores of the most saturated lines. We attribute this either to an incorrect background subtraction ${ }^{3}$ or to less reliable models of atmospheres for cool giants

\footnotetext{
${ }^{3}$ Since we used the image slicer, the flux-per-pixel is quite low in our spectra, which may complicate the background light subtraction procedure.
}

leading to an imperfect treatment of the line cores. In Santos et al. (2002) this problem was not found for cool dwarfs, although they did not study any star with $T_{\text {eff }}$ lower than $5200 \mathrm{~K}$. In any case, since the Be abundances were estimated from the redder BeII line (not affected by saturated lines), a few tests have shown that this does not change any of the results presented here.

Alternatively, this veiling could be related to the solar missing opacity problem (Balachandran \& Bell 1998). However, it is not clear if one can extrapolate this issue for the domain of cool giant stars. Also, it may be that this missing opacity is not needed even for the Sun (e.g., Allende Prieto \& Lambert 2000).

We tested the sensitivity of our derived Be abundances to changes in surface gravity $( \pm 0.5 \mathrm{dex})$, effective temperature $( \pm 150 \mathrm{~K})$, and macro-turbulence $\left( \pm 2 \mathrm{~km} \mathrm{~s}^{-1}\right)$. The results show that for such low Be levels no changes are found. In all cases, the final abundances are always compatible with no detection of Be. Other possible sources of errors in the derived Be abundances are well discussed in García López (1995) and Santos et al. (2004b). We refer to these works for further details.

\section{Results and discussion}

\subsection{Be is fully depleted}

The main results from the present observations are reported in Table 2, where in addition to the beryllium abundances $A(\mathrm{Be})$, $[\mathrm{O} / \mathrm{H}],[\mathrm{Mn} / \mathrm{H}]$, and $[\mathrm{Tm} / \mathrm{H}]$ are also presented for the Li-rich giants and the Li-normal giants.

A first important and striking result concerns the very low values of $A(\mathrm{Be})$. Beryllium is essentially absent in the 

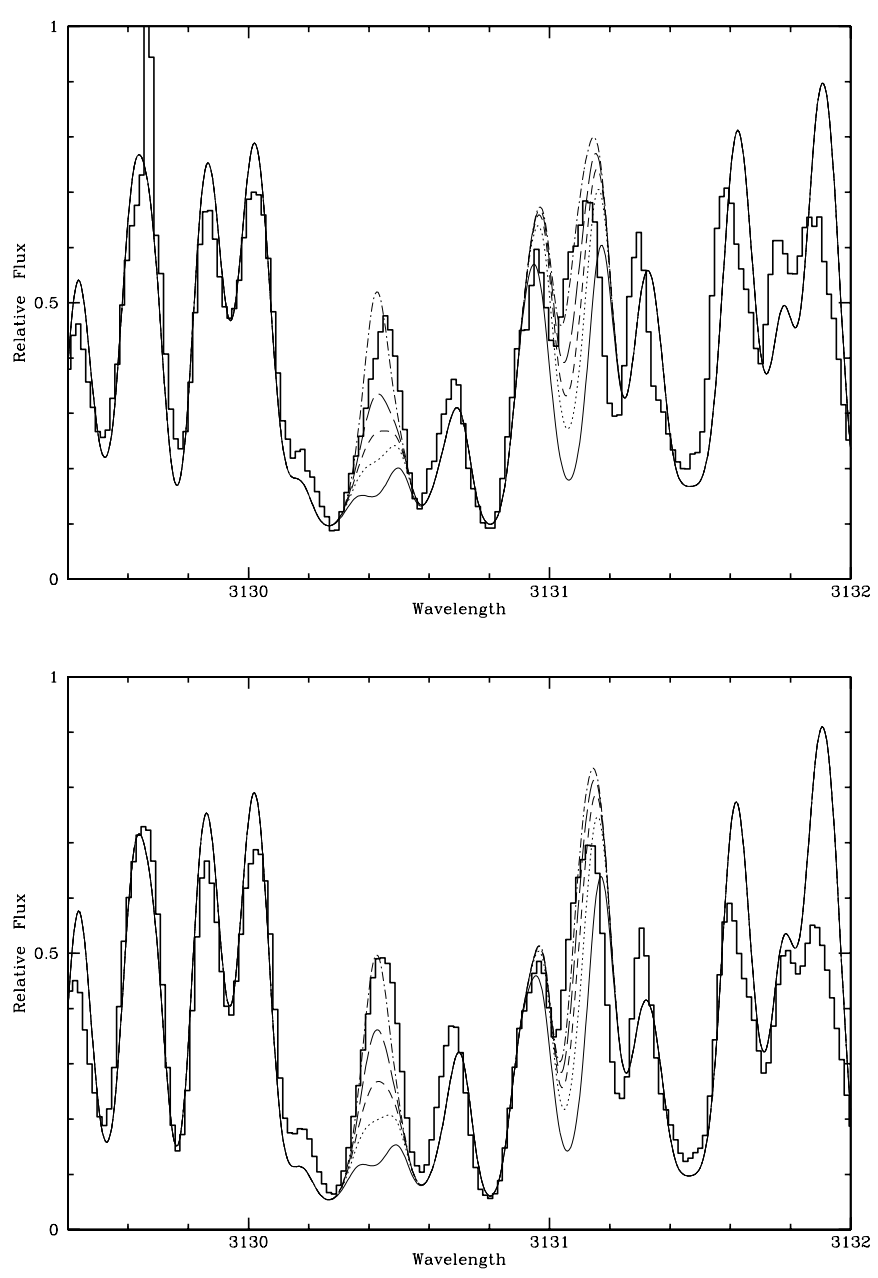

Fig. 3. Example of spectral syntheses (continuous lines) and observed spectra (histogram) in the Be II line region for 2 Li-rich stars HD 19745 and HD 95799. In all panels, the lower to upper syntheses correspond to $A(\mathrm{Be})$ of $0.0,-1.0,-1.5,-2.0$, and -5.0 .

atmosphere of all stars presented in Table 2, regardless of their normal or lithium-rich behavior. In Fig. 3 we show the spectral synthesis for two Li-rich and for two Li-normal giants. Given the quality of the spectral syntheses seen in Fig. 3 we can promptly see that our linelist fits the entire region around the Be II well, which excludes a poor spectral synthesis as an explanation of the low values for $A(\mathrm{Be})$.

The fact that no Be was found in the Li-rich stars is intriguing. According to classical stellar evolution models, Li and $\mathrm{Be}$ depletions are not expected to take place during the main-sequence, since the survival zones for both elements are deeper than the depth of the convective zones of F-G dwarfs (e.g., review by Deliyannis et al. 2000). During the post-mainsequence evolution, as the convective zone deepens, the stellar material "rich" in light elements $(\mathrm{Li}, \mathrm{Be}$, and B) is mixed with material with no such elements, so the surface content is decreased by dilution. Boesgaard et al. (1977) compared both the $\mathrm{Li}$ and $\mathrm{Be}$ abundances of four giants in the Hyades to the abundance values measured among the dwarfs in the same cluster. They found that the giants are deficient in Li by a factor of 110 , whereas Be is depleted by a factor between 20-70 compared to the dwarfs in cluster.
Although the standard models can provide some insight into the light element depletion inside the stars, it is a known fact that they are not able to explain most of the observed behaviors of Li and Be (e.g. Stephens et al. 1997), which leads to exploring alternative depletion mechanisms acting already on the main-sequence. For instance, simultaneous $\mathrm{Li}$ and $\mathrm{Be}$ depletion is foreseen in models with diffusion for stars cooler than about $6600 \mathrm{~K}$, whereas models based on mass loss predict a flat $\mathrm{Be}$ versus $\mathrm{Li}$ distribution. In models with slow mixing, Li depletion should be more efficient than $\mathrm{Be}$, but correlation between $\mathrm{Be}$ and $\mathrm{Li}$ is predicted (see review by Delyannis et al. 2000, and references therein).

Recent observational work devoted to the measurement of $\mathrm{Li}$ and $\mathrm{Be}$ abundances for main-sequence dwarfs have shown that both elements are indeed depleted during the mainsequence (Stephens et al. 1997; Santos et al. 2002, 2004a). In particular, Santos et al. (2002, 2004a) have shown that Be abundances (and depletion rates) seem to be a function of the effective temperature with a maximum close to the meteoritic value $(A(\mathrm{Be})=1.42$, Anders \& Grevesse 1989$)$ near $T_{\text {eff }} \sim 6100 \mathrm{~K}$, decreasing both towards higher (Be-gap for $\mathrm{F}$ stars) and lower temperatures. A similar trend is also observed for the Li abundances. Although Li and Be depletion rates do not necessarily happen at the same rate (Santos et al. 2002, 2004a; although see Boesgaard et al. 2001, for a counter example of correlated Li and Be depletion), that i) both elements are already depleted on the main-sequence and that ii) as a general rule, Li depletion takes place much faster than Be both seem undeniable.

For stars on the blue side of the Li gap (i.e., $T_{\text {eff }} \gtrsim 7000 \mathrm{~K}$ or $M \gtrsim 1.5 M_{\odot}$ ) little information on the light element abundances is available. In a series of papers Burkhart \& Coupry (1997, 2000a,b) measured the Li abundances of normal A- and Am-stars (slowly rotating magnetic A stars) in different open clusters. They show that, for each studied cluster, the Li abundances of the normal A stars set the maximum value for the cluster. Normal A stars are not believed to deplete Li whether during the PMS or during the MS phase; in addition, as fast rotators they are supposed to be less affected by any depletion caused by settling or separation process. Therefore, giant stars in this mass regime had probably not experienced Li depletion during their PMS and MS phase. In conclusion, the complete absence of $\mathrm{Be}$ in the atmospheres of the Li-rich giants in our sample cannot be ascribed to their MS depletion histories, because i) a combined depletion of both elements is expected to occur and ii) Li is destroyed faster than Be. For more massive stars on the blue side of the Li gap, no substantial depletion is expected.

\subsection{The origin of the $\mathrm{Li}$ in the Li-rich giants}

The possible origin of the $\mathrm{Li}$ in the Li-rich giants has been widely discussed in the literature for many years now (see e.g. Charbonnel \& Balachandran 2000; de la Reza 2000, and references therein). So far, none of the proposed scenarios have been found to explain the existence of the Li-rich giants. Nonetheless, the internal mixing and convective scenarios seem appealing given our results for the Be abundances. These 

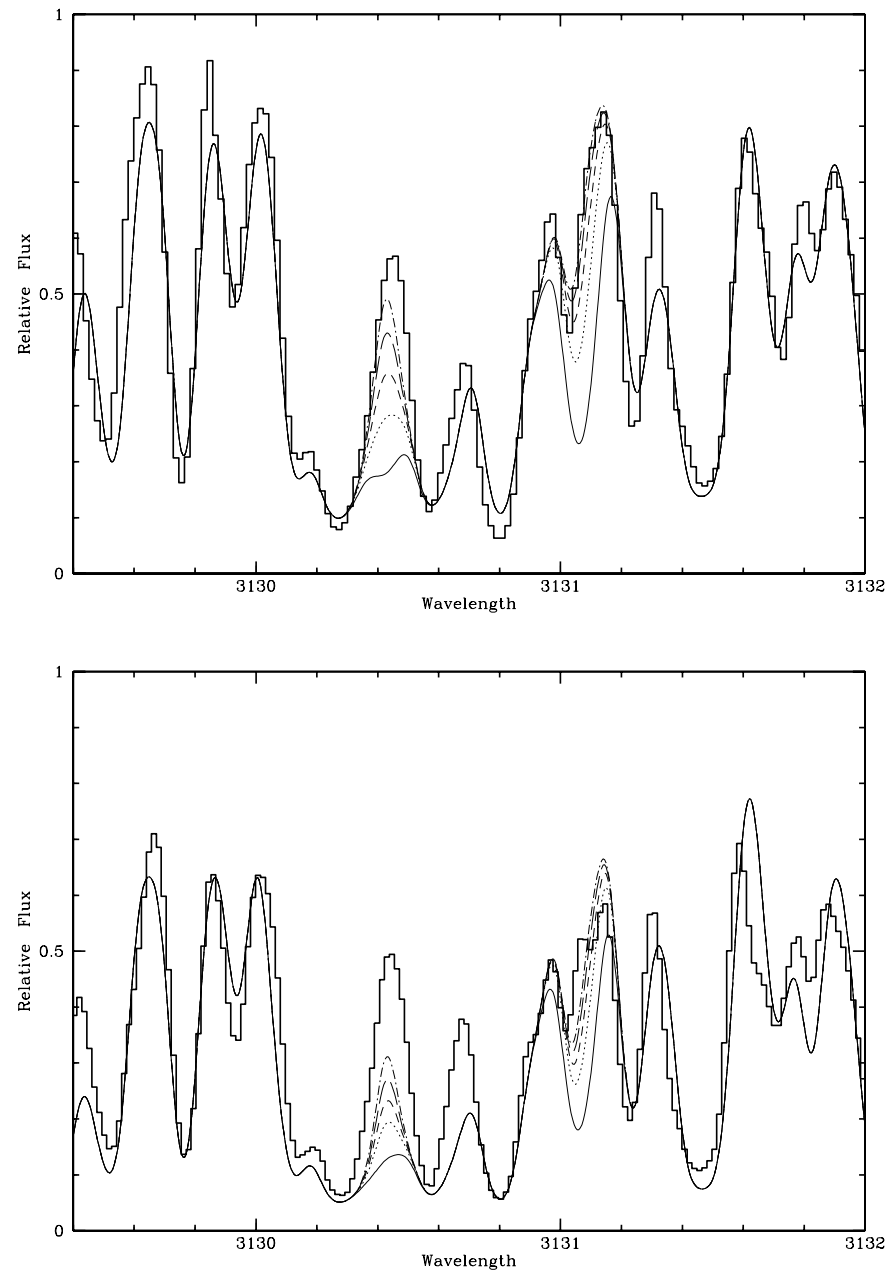

Fig. 4. Same as Fig. 3 for the 2 Li-normal stars HD 360 and HD 126217.

scenarios are based on the Cameron \& Fowler (1971) process in which ${ }^{7} \mathrm{Be}$ is produced in the hot internal layers corresponding to the H-burning zone through the reaction ${ }^{3} \mathrm{He}(\alpha, \gamma){ }^{7} \mathrm{Be}$. For giants with masses between 3-6 $M_{\odot}$, this is close to the the internal base of the convective zone, therefore ${ }^{7} \mathrm{Be}$ produced by the reaction above is promptly transported to the outer layers where it is finally transformed into ${ }^{7} \mathrm{Li}$ by the reaction ${ }^{7} \mathrm{Be}\left(\mathrm{e}^{-}, v\right)^{7} \mathrm{Li}$. This mechanism is known as the Hot Bottom Burning proposed by Sackmann \& Boothroyd (1992). For less massive giants $<2.5 M_{\odot}$ the internal base of the convective zone does not reach the H-burning zone, thus the ${ }^{3} \mathrm{He}$ from the envelope cannot be converted into Be. In order to solve this problem, Sackmann \& Boothroyd (1999) proposed the Cool Bottom Processing (CBP) model, in which an ad-hoc twostream conveyor-belt is responsible for circulating the ${ }^{3} \mathrm{He}$-rich envelope of the red giant to the $\mathrm{H}$-burning zone producing fresh ${ }^{7} \mathrm{Be}$, which is then transported to the external layers and transformed into ${ }^{7} \mathrm{Li}$ again by the reaction ${ }^{7} \mathrm{Be}\left(\mathrm{e}^{-}, v\right)^{7} \mathrm{Li}$.

On the one hand, the CBP model described above seems to qualitatively explain our results. According to Sackmann \& Boothroyd (1999), if the deep mixing is continuous and longlived, then the star must show $\mathrm{Li}$ enrichment due to fresh $\mathrm{Li}$ brought to the surface and be completely devoid of beryllium and boron. Charbonnel \& Balachandran (2000), on the other hand argue that, if such a long-lived mixing episode occurs, this must be reflected in the values of ${ }^{12} \mathrm{C} /{ }^{13} \mathrm{C}$, which should continuously decrease along the $\mathrm{RGB}$, reaching values much lower than the standard values that result from the first dredgeup, which is not observed.

In summary, the complete absence of $\mathrm{Be}$ in the atmosphere of the Li-rich giants suggests that it is very unlikely (from the physical point of view) that the $\mathrm{Li}$ we see is that preserved from the main-sequence phase. Our results strongly favor the BCP mechanism proposed by Sackmann \& Boothroyd (1999) as the source of Li enrichment. However, as pointed out by Charbonnel \& Balachandran (2000), this scenario still presents some caveats. A similar conclusion was drawn by Castilho et al. (1999), who derived $A(\mathrm{Be})$ for three giants, two Li-rich (HD 787 and HD 148650), and another Li-normal (HD 220321). They found that these stars had depleted more the $90 \%$ of their Be (assuming an initial $A(\mathrm{Be})=1.42$ ) regardless of their Li-rich or Li-normal nature. All of the three giants studied by Castilho et al. (1999) were re-observed in this work. Although the qualitative results are the same, the absolute values of abundances are different, while Castilho et al. still find some Be in the atmosphere of these 3 stars, we found no trace of this element. A possible explanation might come from the difference in resolution and sensitivity of the spectra collected with CASPEC and those taken to UVES, which has roughly 2 times more resolving power and an $S / N$ twice as good. Another more plausible explanation is a difference in the linelists. Castilho et al. added an ad-hoc Fe I at $3130.995 \AA$ in order to fit the Be region of two dwarfs, $\alpha$ Cen $\mathrm{A}$ and $\alpha$ Cen B, used as reference stars. In our case, we found it more appropriate to calibrate our oscillator strength using Arcturus as a reference star. As discussed in Sect. 3.2, this procedure lead to a good fit without any need to include extra iron lines.

\subsection{Engulfment episode?}

The observational fact that most of the Li-rich giants are associated with IRAS sources led to de la Reza et al. $(1996,1997)$ to propose a scenario linking the Li excess to infrared properties. They suggest that the $\mathrm{Li}$ enrichment produced by an internal $\mathrm{Li}$ production mechanism based on the Cameron-Fowler mechanism will also trigger the formation of a circumstellar shell. The mass loss suddenly stops and the CS detaches making the star move in the IRAS color-color diagram from zone I to II, II to III, and finally back to I again in what they call the Li-cycle. (see e.g., Fig. 1 of de la Reza et al. 1997). In parallel with the expansion of the circumstellar shell, fresh $\mathrm{Li}$ is destroyed by the very same mechanism which created it, namely, the deep mixing. Thus the actual location of the star in the IRAS color-color diagram depends both on the difference between the time-scale of the expansion of the circumstellar shell and on the time-scale for Li depletion. In spite of being attractive, this scenario does not explain how the deep mixing and mass loss phenomena are connected. Also, precise predictions for the photospheric abundance pattern $\left({ }^{7} \mathrm{Li},{ }^{9} \mathrm{Be},{ }^{12} \mathrm{C} /{ }^{13} \mathrm{C}\right.$, for instance) as a function of time are not given. 

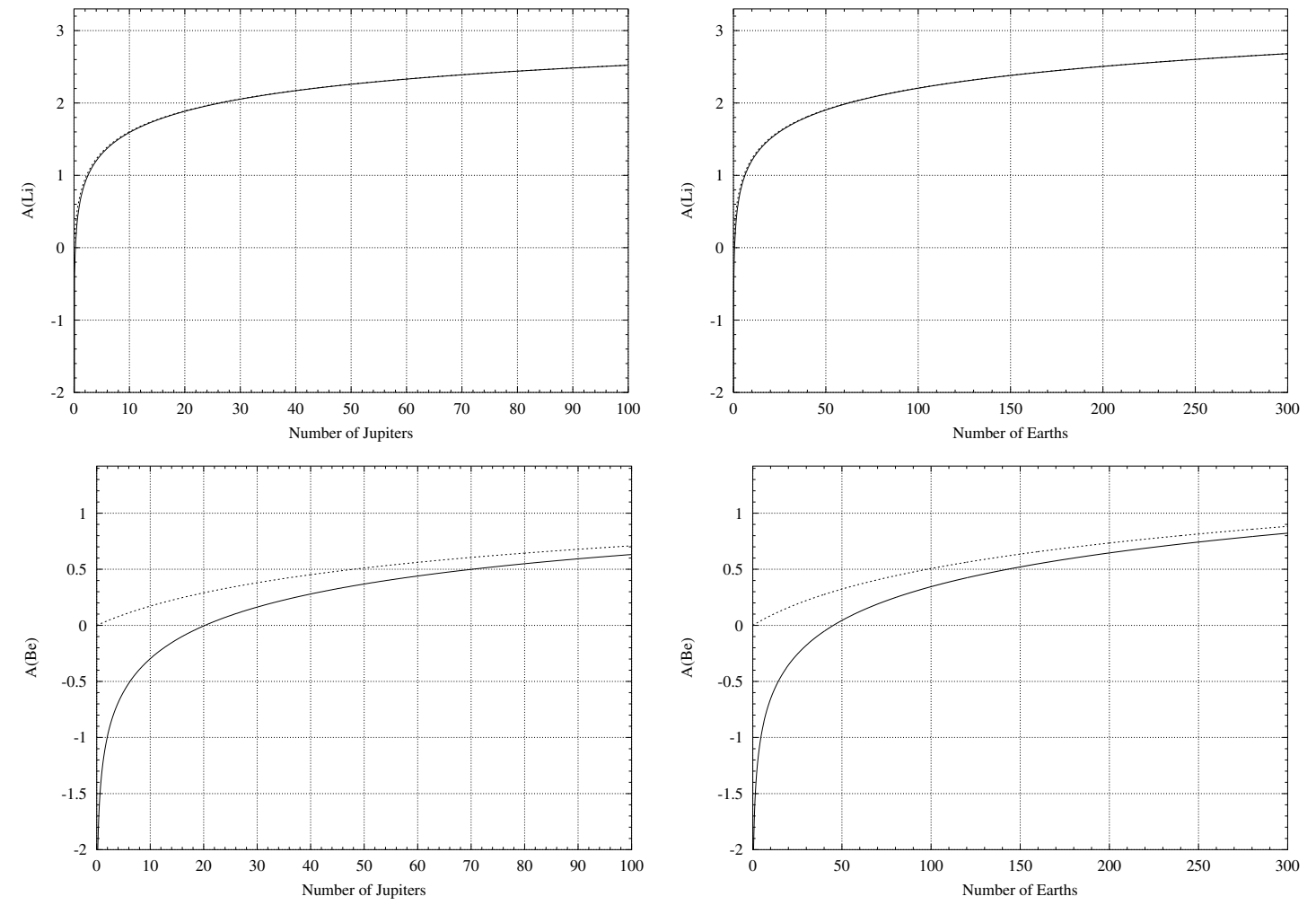

Fig. 5. $\mathrm{Li}$ and $\mathrm{Be}$ abundance curves as a function of the mass and nature of the accreted material. Left top panel shows Li enrichment for two initial abundances $A(\mathrm{Li})=-10$ (solid line) and $A(\mathrm{Li})=0.0$ (dotted line) as a function of the number of Jupiters engulfed. In the bottom panel similar curves are shown for the Be enrichment. Right: Li and Be enrichment curves for chondritic-like engulfed material given in Earth mass. In all cases, a convective zone of $0.5 M_{\odot}$ was assumed. See text for more details.

Siess \& Livio (1999) studied in detail the implications of the engulfment of a giant planet or a brown dwarf by a giant star. They show that such an event will be accompanied by several observational signatures, such as production and posterior detachment of a circumstellar shell, light element enrichment, spin-up, and X-ray activity enhancement. The circumstellar shell and its detachment occur as a consequence of the thermal pulse caused by the accreted material (i.e., engulfed object). Their calculations show that the higher the accretion rate, the greater the increase in the mass-loss rate; while the more evolved the star, the easier it is to remove material from its surface due to a lower surface gravity. Thus the Li-cycle as proposed by de la Reza et al. $(1996,1997)$ is naturally explained as the consequence of the engulfment phenomena.

Another important observational signature of an engulfment episode is the light element enrichment mainly of ${ }^{7} \mathrm{Li}$ and ${ }^{9}$ Be. Siess \& Livio (1999) already acknowledge the fact that in order to explain $\mathrm{Li}$ abundances as high as $A(\mathrm{Li}) \sim 2.5$ based on their engulfment scenario: either i) the amount of mass accreted was unlikely high (i.e., several planets) or ii) the Li content of a brown dwarf is extremely high (Li mass fraction would be typically 10 times higher in the brown dwarf than in the stellar envelope). In order to overcome this problem, they claim that accreted material will also trigger the cool bottom burning discussed above, which will help to increase the Li content.
In light of the total absence of $\mathrm{Be}$ in these stars (cf. Sect. 4.1), can the hypothesis of Li enrichment due to accretion of an external Li-rich material be ruled out? In Fig. 5 we show the final abundances of $\mathrm{Li}$ and $\mathrm{Be}$ expected for a giant star with a convective zone of $0.5 M_{\odot}$ and for two initial $\mathrm{Li}$ and Be abundances as a function of the accreted mass. The accreted material is either of Jupiter- (left) or chondritic-like nature. In the case of Jupiter-like material, meteoritic abundances for $\mathrm{Li}$ and Be have been assumed (Anders \& Grevesse 1989) whereas for the chodritic-like material $\mathrm{Li}$ - and Be-to-iron ratios have been assumed to be meteoritic (Anders \& Grevesse 1989) and the mass fraction of iron to be $19 \%$.

Looking at Fig. 5, we see that in order to match the Li abundance of a very Li-rich giant $(A(\mathrm{Li}) \sim 2.5)$, an ordinary postdiluted giant has to accrete $\sim 100$ Jupiters, i.e., $0.1 M_{\odot}$, regardless of the initial $\mathrm{Li}$ abundances $(A(\mathrm{Li})=-10 A(\mathrm{Li})=0.0)$. As far as the Be is concerned, for an accreted mass of $0.1 M_{\odot}$, the final $A(\mathrm{Be})$ is expected to be $0.6-0.7$ depending on the initial Be content. For an accreted mass of 10 Jupiters, the Li and Be abundances are expected to be 1.5 and -0.3 , respectively. Now, if the composition of the accreted material is chondriticlike, then we would need about 40 Earth-masses of chondriticlike material in order to enrich the envelope of giant star to the level of $A(\mathrm{Li}) \sim 2.0$, which would bring the $A(\mathrm{Be})$ to about 0.0. It is interesting to note, however, that if close-in giant 
planets are super-metallic (e.g. due to hydrogen evaporation Vidal-Madjar et al. 2003), then a lower, and eventually reasonable, number of accreted Jupiter-like planets would be needed for the star to achieve high Li-abundance.

In Fig. 3 we show the spectra obtained for four of the program stars overplotted with the spectral synthesis for different Be abundances, namely, $A(\mathrm{Be})=0.0,-1.0,-1.5,-2.0,-5.0$ (no $\mathrm{Be}$ ). We see that an $A(\mathrm{Be})$ abundance of $\sim 0.0$ (corresponding to a $A(\mathrm{Li})=1.5$ and an accreted mass of 10 Jupiters) would be easily detected in our observations according to Fig. 3. Thus, our newly derived $A(\mathrm{Be})$ show that it is unlikely that the Lirich giants were enriched via engulfment episodes. Moreover, the absence of Be suggest that a Cameron-Fowler mechanism is behind the Li enrichment in these stars. However, the IR excess observed for a few Li-rich and Li-normal stars still remain to be fully explained.

The typical radius of a solar mass red giant is of about $70 R_{\odot}$ or $\sim 0.3 \mathrm{AU}$, which corresponds to roughly the size of the orbit of Mercury, i.e., to an orbital period of about 60-100 days. Given the orbital period distribution of the known extrasolar planets, we see that $\sim 30$ per cent of the known extra-solar planets are going to sufer the inexorable fate of being engulfed by their own sun when it reaches the red giant branch. Therefore engulfment does occur. Rather than ruling out the engulfment phenomenon itself, our results only say that, given the ${ }^{7} \mathrm{Li}$ and ${ }^{9} \mathrm{Be}$ abundances shown here, engulfment as the main source of $\mathrm{Li}$ enrichment is ruled out.

Finally, throughout the paper we have argued that it is very likely that the Cameron-Fowler mechanism is responsible for the Li enrichment observed in some giant stars; however, what triggers the Cameron-Fowler mechanism is not clear. Also, why is it ignited in some stars and not in others? These are important open questions beyond the scope of this paper.

\section{Conclusions}

In this paper we have analyzed the Be abundances for a sample of Li-rich giant stars with the aim of testing the engulfment hypothesis suggested by Siess \& Livio (1999). The main results obtained can be outlined as follows:

- No Be was found in any of the observed stars, regardless of their nature Li-rich or Li-normal. The absence of Be is real and it is not an artifact of the spectral syntheses as shown by the good fit achieved for Arcturus (Fig. 2) and for the Li-rich and Li-normal giants (Fig. 3).

- The Cameron-Fowler mechanism is favored. The presence of $\mathrm{Li}$ and the absence of Be strongly suggests that the $\mathrm{Li}$ enrichment is probably the result of the dredge-up of fresh Li produced in the interior of these stars by the extramixing mechanism proposed by Sackmann \& Boothroyd (1999). However, the fact that the model fails to predict the observed ${ }^{12} \mathrm{C} /{ }^{13} \mathrm{C}$ indicates that it still requires improvement.

- Engulfment cannot explain the presence of $\mathrm{Li}$ and absence of Be. Using simple dilution arguments, we show that the accreted mass necessary to produce the Li-rich enrichment observed in the Li-rich giants would also produce a Be enrichment detectable by our observations. Thus our newly derived Be abundances rule out engulfment as the sole source of the Li observed in the Li-rich giants.

Acknowledgements. We would like to warmly thank the Paranal Observatory staff, especially the UT2/Kueyen service mode observers, for carrying out these observations in an efficient and competent way. Support from Fundação para a Ciência e Tecnologia (Portugal) to N.C.S. in the form of a scholarship is gratefully acknowledged. J.D.N.Jr. acknowledges the CNPq grant PROFIX 540461/016. J.R.DM has been supported by continuous grants from the CNPq Brazilian Agency. We are grateful to Dr. Guillermo Gonzalez who kindly provided the Arcturus spectrum used in this work. We thank the anonymous referee whose comments helped to improve the paper. Finally, we thank Mr. J. Adams for his careful revision of the text.

\section{References}

Allende Prieto, C., \& Lambert, D. L. 2000, AJ, 119, 2445

Alexander, J. B. 1967, The Observatory, 87, 238

Alonso, A., Arribas, S., \& Martínez-Roger, C. 1999, A\&AS, 140, 261

Anders, E., \& Grevesse, N. 1989, Geochim. Cosmochim. Acta, 53, 197

Balachandran, S. C., \& Bell, R. A. 1998, Nature, 392, 791

Balachandran, S. C., Henry, G., Fekel, F. C., \& Uitebroek, H. 2000, ApJ, 542, 978

Bertelli, G., Bressan, A., Chiosi, C., Fagotto, F., \& Nasi, E. 1994, A\&AS, 106, 275

Boesgaard, A. M., Heacox, W. D., \& Conti, P. S. 1977, ApJ, 214, 124

Boesgaard, A. M., Deliyannis, C. P., King, J. R., \& Stephens, A. 2001, ApJ, 553, 754

Brown, J. A., Sneden, C., Lambert, D. L., \& Dutchover, E. J. 1989, ApJS, 71, 293

Burkhart, C., \& Coupry, M. F. 1997, A\&A, 318, 870

Burkhart, C., \& Coupry, M. F. 2000a, A\&A, 338, 1073

Burkhart, C., \& Coupry, M. F. 2000b, A\&A, 354, 216

Cameron, A. G. W., \& Fowler, W. A. 1971, ApJ, 164, 111

Castilho, B. V., Spite, F., Barbuy, B., et al. 1999, A\&A, 345, 249

Castilho, B. V., Gregorio-Hetem, J., Spite, F., Barbuy, B., \& Spite, M. 2000, A\&A, 364, 674

Charbonnel, C., \& Balachandran, S. C. 2000, A\&A, 359, 563

Chmielewski, Y., Müller, E. A., \& Brault, J. W. 1975, A\&A, 42, 37

de la Reza, R., \& da Silva, L. 1995, ApJ, 439, 917

Dekker, H., D’Odorico, S., Kaufer A., Delabre, B., \& Kotzlowski, H. 2000, Proc. SPIE, 4008, 534

Dekker, H., Nissen, P. E., Kaufer, A., et al. 2002, Proc. SPIE, 4842, 28

de La Reza, R. 2000, IAU Symp., 198, 310

de La Reza, R., Drake, N. A., \& da Silva, L. 1996, ApJ, 456, L115

de La Reza, R., Drake, N. A., da Silva, L., Torres, C. A. O., \& Martin, E. L. 1997, ApJ, 482, L77

de Laverny, P., do Nascimiento, J.D. Jr., Lèbre, A., \& de Medeiros, J. R. 2003, A\&A, 410, 937

De Medeiros, J. R., Lebre, A., de Garcia Maia, M. R., \& Monier, R. 1997, A\&A, 321, L37

De Medeiros, J. R., \& Mayor, M. 1999, A\&AS, 139, 433

Deliyannis, C. P., Pinsonneault, M. H., \& Charbonnel, C. 2000, IAU Symp., 198, 61

Fekel, F. C., \& Balachandran, S. 1993, ApJ, 403, 708

Feltzing, S., \& Gustafsson, B. 1998, A\&AS, 129, 237

Flower, P. J. 1996, ApJ, 469, 355

García López, R. J., Severino, G., \& Gomez, M. T. 1995, A\&A, 297, 787

Gratton, R. G., \& D’Antona, F. 1989, A\&A, 215, 66 
Hauck, B., \& Mermilliod, J.-C. 1998, A\&AS, 129, 431

Houdashelt, M. L., Bell, R. A., \& Sweigart, A. V. 2000, AJ, 119, 1448 Iben, I., Jr. 1967, ApJ, 424, 1005

Israelian, G., Santos, N. C., Mayor, M., \& Rebolo, R. 2001, Nature, 411,163

Israelian, G., Santos, N. C., Mayor, M., \& Rebolo, R. 2003, A\&A, 405,753

Jasniewicz, G., Parthasarathy, M., de Laverny, P., \& Thévenin, F. 1999, A\&A, 342, 831

Kurucz, R. L. 1883, CD-ROMs, ATLAS9 Stellar Atmospheres Programs and $2 \mathrm{~km} \mathrm{~s}^{-1}$ Grid (Cambridge: Smithsonian Astrophys. Obs.)

Luck, R. E. 1994, ApJS, 91, 309

Nissen, P. E., Hoeg, E., \& Schuster, W. J. 1997, in Proceedings of the ESA Symposium Hipparcos - Venice 97, ESA SP-402

Perryman, M. A. C., Lindegren, L., Kovalevsky, J., et al. 1997, A\&A 323, 49

Peterson, R. C., Dalle Ore, C. M., \& Kurucz, R. L. 1973, ApJ, 404, 333
Pinsonneault, M. H., Kawaler, S. D., \& Demarque, P. 1990, ApJS, 74, 501

Primas, F., Duncan, D. K., Pinsonneault, M. H., Deliyannis, C. P., \& Thorburn, J. A. 1997, ApJ, 480, 784

Sackmann, I.-J., \& Boothroyd, A. I. 1992, ApJ, 392, L71

Sackmann, I.-J., \& Boothroyd, A. I. 1999, ApJ, 510, 217

Santos, N. C., García López, R. J., Israelian, G., et al. 2002, A\&A, 386,1028

Santos, N. C., Israelian, G., Randich, S., García López, R. J., \& Rebolo, R. 2004a, A\&A, 425, 1013

Santos, N. C., Israelian, G., García López, R. J., et al. 2004b, A\&A, 427,1085

Siess, L., \& Livio, M. 1999, MNRAS, 308, 1133

Snenden, C. 1973, Ph.D. Thesis, University of Texas

Stephens, A., Boesgaard, A. M., King, J. R., \& Deliyannis, C. P. 1997, ApJ, 491, 339

Vidal-Madjar, A., Lecavelier des Etangs, A., Dśert, J.-M., et al. 2003, Nature, 422, 143 